\title{
DEVELOPMENT OF SPECIAL EXPLOSIVE CHARGES FOR THE RECONSTRUCTION OF UNSTABLE DAMAGED BUILDINGS
}

\author{
STANISLAV LICHOROBIEC, MIROSLAV MYNARZ \& PETR LEPÍK \\ VŠB - Technical University of Ostrava, Faculty of Safety Engineering, Czech Republic
}

\begin{abstract}
Buildings or properties seriously damaged due to detonation of explosives, gas explosions, technological accidents, and other emergency's threaten their surroundings with their instability. Their damage is usually so significant that no repair or reconstruction is ever considered, demolition is inevitable. In such hazardous situations, use of special charges could be the possible solution for a safe disposal of damaged and statically unstable structures. Use of this emergency pyrotechnic instrument could eliminate or considerably reduce the possibility of health hazards of intervening people, rescue equipment and surrounding properties during the disposal process of extremely damaged buildings in a very short time, mainly in neighbourhoods. A System of special sequentially timed charges use the increase of efficiency of external charges and cover with water-bags together with the increase of efficiency of the whole system due to their timing. Developed charges increase the operability and safety of rescue units of IRS (Integrated Rescue System).
\end{abstract}

Keywords: special charge, unstable building, damage, shock wave, semtex.

\section{INTRODUCTION}

For members of the Fire Rescue Service, it is sometimes necessary to demolish buildings that are damaged due to a serious incident which cause irreversible damage. They move in the immediate vicinity of unstable walls or they use heavy wreckers for demolition. The intended explosion of the block of flats in Frenštát pod Radhoštěm (Czech Rep.) could be used as an example: due to blast wave impact its walls moved, the building became unstable and its demolition using wrecking equipment was very hazardous, including serious danger for intervening people.

Use of a developed system of special directed explosive charge enables demolition of the building without exposure of persons or used techniques and neither surrounding buildings are threatened by such destructive action.

After some incidents as described above, the strength of used building materials and structure elements could decrease up to $30-60 \%$. These values are obtained only by expert opinion and experience of a structural engineer. Use of an explosive charge for the predicted weakest part of damaged building is also connected with knowledge of the effect of a developed explosive charge depending on resistance of damaged structural elements.

\section{ACCELERATING OF MASS THROUGH EXPLOSION}

At the explosion of disruptive explosive with minimum detonation velocity of $6000 \mathrm{~m} / \mathrm{s}$, originated gases expand essentially in all directions and they copy shapes of the explosive charge. The higher the detonation velocity of the explosive charge, the more distinctly the directivity effects become evident. Gases originated at the explosion expand faster from places with a higher concentration of the explosive than from places with a lower concentration. Creation of a suitable cavity in a disruptive explosive charge enables regulating the flow of gases so that they can join together to the compact flow. The flow of 
gases with very high velocity is obtained which then corresponds to a huge accumulation of energy.

Density of the flow of gases is still not yet sufficient enough to cause a strong destruction of material. Its density and efficiency could be increased by placing a metal insert to the cavity in the front part of the explosive charge, or by filling the cavity e.g. with water. Incompressibility is one of waters characteristics as is water consistency which also increases the efficiency of such regulated explosive charges. The explosive charges modified in this way are placed to the wall of the statically damaged building and therefore cause the destruction of the structure by its explosion effect and water directed jet [2]-[4].

Detonation pressure is the highest pressure of explosion products in the detonation wave in the case that it follows the ideal gas law according to the state eqn (1):

$$
p \cdot V=n \cdot M \cdot R \cdot T ; \quad p=n \cdot M \cdot R \cdot T / V
$$

where: $\mathrm{p}$ - pressure [MPa], $\mathrm{V}$ - volume $\left[\mathrm{m}^{3}\right], \mathrm{n}$ - number of moles in $1 \mathrm{~kg}$ of explosive charge, $\mathrm{R}$ - gas constant [-], $\mathrm{T}$ - temperature $[\mathrm{K}], \mathrm{M}$ - explosive charge weight $[\mathrm{kg}]$.

Some of the best-known examples of mass acceleration in our context using explosive charge are a shot, exploitation of minerals and rocks or breaking ice on icebound water surface. Optimal comparison for water acceleration with the help of the explosive charge could be realized through a so-called Gurney model used for derivation of velocity of fragments accelerated by an explosion [5]. Following assumptions are the base of this model [3]:

1. specific amounts of energy related to the unit of mass releases by explosion of given explosive; this energy divides to accelerated of inert matter in the form of kinetic energy and to energy passes to gas products of explosion,

2. originated gas products contain spatially uniform density and linear 1D velocity profile in spatial dimensions of system.

As part of a total energy that is available for mass acceleration it is expressed by the value in dimensions of velocity $\left(\mathrm{km} \cdot \mathrm{s}^{-1}\right)$, characteristic for each explosive, or more precisely for its density, and it is defined by Gurney velocity $(2 \cdot E)^{1 / 2}$. The Cylinder test is used for exact determination of this value.

Depending on used explosive charge, this value could be calculated from defined detonation velocity " $D$ " of concerned explosive charge using the eqn (2):

$$
\sqrt{2 \cdot E}=\frac{D}{2,97}
$$

where: $\sqrt{2 \cdot \mathrm{E}}-$ Gurney velocity $\left(\mathrm{km} \cdot \mathrm{s}^{-1}\right), \mathrm{D}-$ detonation velocity $\left(\mathrm{km} \cdot \mathrm{s}^{-1}\right), 2,97-$ dimensionless coefficient.

\section{SYSTEM OF SPECIAL SEQUENTIALLY TIMED EXPLOSIVE CHARGES}

The system of special sequentially timed directed explosive charges is the alternative method of explosive charges which was developed for breaking ice flows in icebound rivers and can also be used for the destruction of damaged buildings. Aforementioned, one of the advantages is the remarkable minimalization of the negative effect of ice fragmentation and the shattering 
of pieces into the air, which is also helpful for the prevention of dangerous wall fragmentation used in damaged buildings.

Concept of technical solution results from the use of exact timed cutting charges in the ice surface. For higher efficiency of explosive to ice surface, explosive charges are tight in their upper part with water-bags specially developed for this purpose. This method is not as effective as explosive charges under the ice, however the power of such cover explosive charges is still higher than in the case of being freely situated on the ice itself.

The main advantage of this developed process is that the water-bags in the form of a filling absorb the back part of the explosion energy above the explosive charge and they also significantly reduce the dispersion of crushed ice pieces and capture frozen silt from going into the surrounding. The exact timing and optimal arrangement of the precisely defined explosive charges on the surface lead to assumed effect at breaking the ice layer and the regulation of the amount of mass of released ice. Considering minimalization of unfavourable effects of realized blasting to surrounding, this system could be used even in a small distance of residential or industrial built-up areas and structures closed to the waterway, especially next to bridges, weirs, moles or icebound watercrafts. This described system consists of three components:

1. Explosive charge: wide scale of industrial or special explosive charges could be used for the removal of ice barriers. Higher brisance of given explosive goes towards finer fragmentation of ice, higher capacity of work increases whole volume of released ice mass.

2. Textile filling water-bags: construction of water-bags was designed considering their sufficient strength at handling, watering and resistance to explosion, see Fig. 1. Their construction enables to put the adjusted explosive charge with the detonator in the pocket at the outer part of the bottom of the bag, to fix the detonator using mounting brackets against pulling out of the charge at arrangement of bags on surface or at their watering.

All parts of the bag including the filling sleeve are made of soft textile material. During explosion, no solid fragments are dispersed. The bags are made with standard volumes of 250 litre and square base with bottom area of c. 1 square meter.

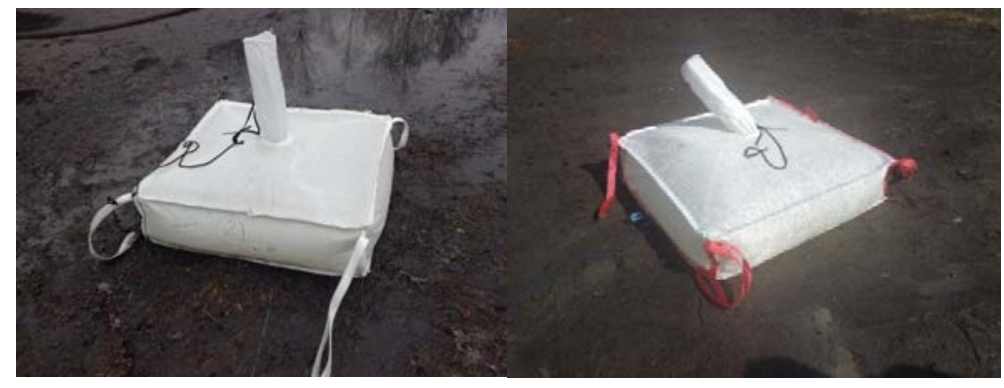

Figure 1: Photo of developed textile bags with volume of 2501 of water.

3. Ignition system: electric or non-electric ignition, as well as timing electronic ignition systems could be used as ignition system. Considering setting of arbitrary sequential ignition times, the system of variable electronic timing is used with an advantage of 
real practical use. Electric ignition was designed for longer times with a lower accuracy of timing between $25 \mathrm{~ms}$ and $75 \mathrm{~ms}$.

\subsection{Seismic measurement of system efficiency}

Seismic measurements of efficiency developed special directed explosive charges were performed by Austin Detonator S.R.O. and Geodyn, spol. S.R.O. The companies used a measuring set of vibrographs - geophones, type Instantel Mini Mate plus, BE 7901, 9146 and 13846. Measurement was carried out in four perpendicular directions to the centre of the first explosive charge, including the measurement of acoustic pressure using a linear microphone with a weighting filter. Geophones were located $10 \mathrm{~m}$ from the centre of the first explosive charge and one more geophone was placed in the distance of $7.5 \mathrm{~m}$ in the direction of sequential timing. Two directed explosive charges with given sequential timing were fired at the same time.

The spatial arrangement of geophones is illustrated in Fig. 2. Results of a particular seismic measurement is given in the following figures. Graphs (Fig. 3) show the outputs from the geophones including mathematical calculation of total energetic efficiency.

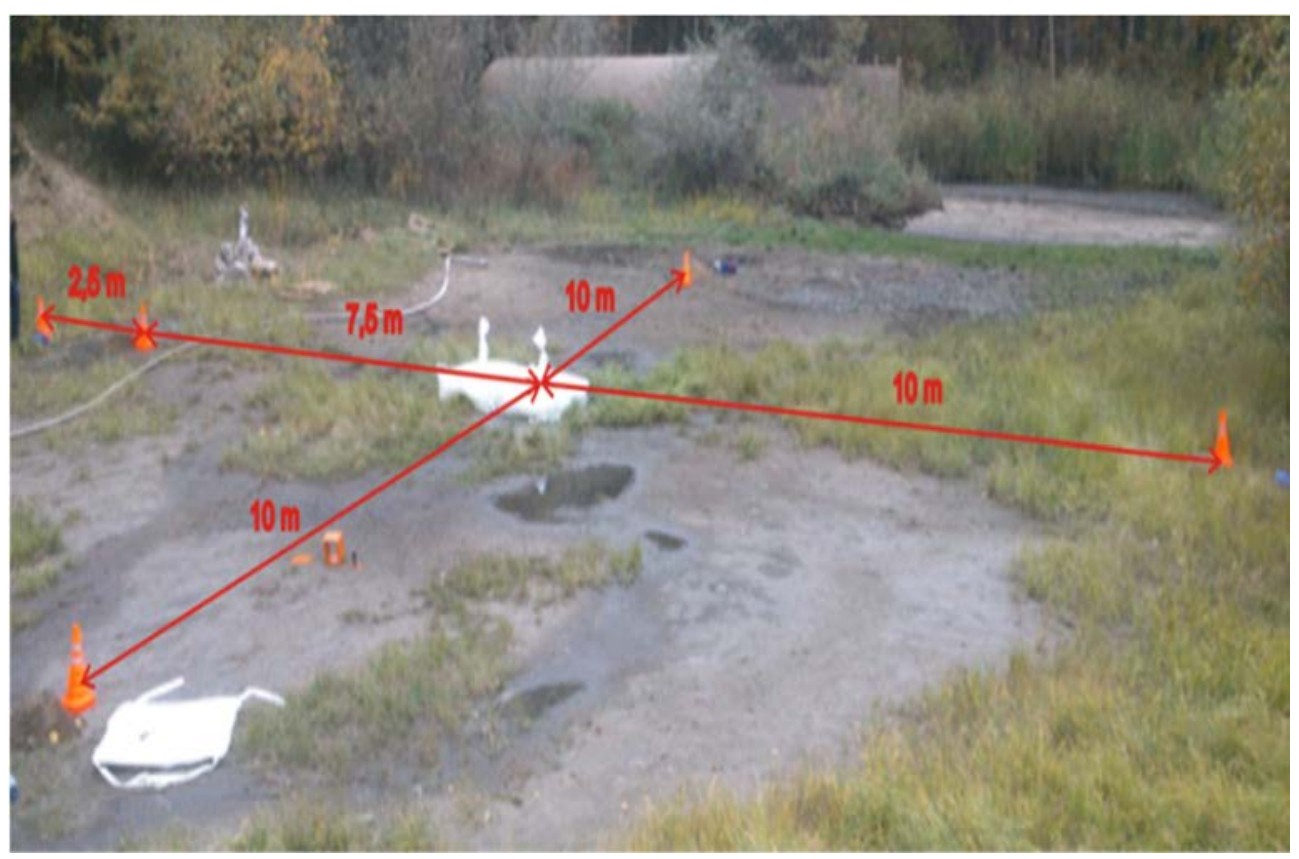

Figure 2: Spatial arrangement of used geophones at seismic measurements. 


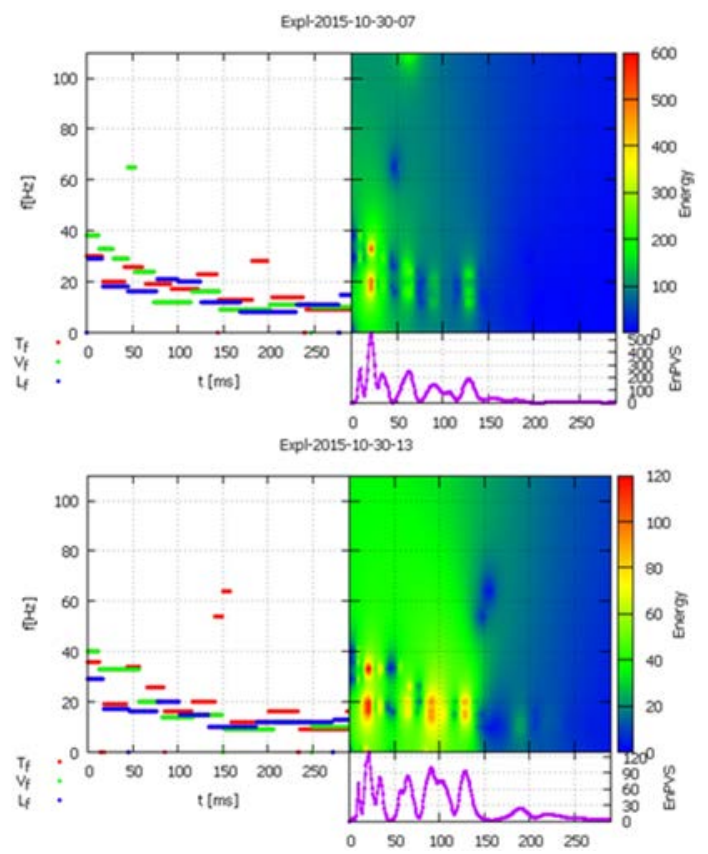

Figure 3: Colour expression of energetic efficiency of particular measurements depending on time and frequency of oscillation of seismic waves.

Total efficient energy of the explosion increased to the value of 19.17 in the first case and to the value of only 8.38 in the second case. With longer distance and higher timing value, it resulted in energy being split. This phenomenon is obvious even when looking at defocusing of the print in the green field in Fig. 3.

\subsection{Practical use of developer system}

This developed system of special sequential explosive charges could be used in many different ways. A significant advantage is a possible preparation on the bank, then transferring to the ice surface and filling it with water. Time sequence of explosion of the explosive charges (see Fig. 4) documents also the minimum danger area at their practical use.
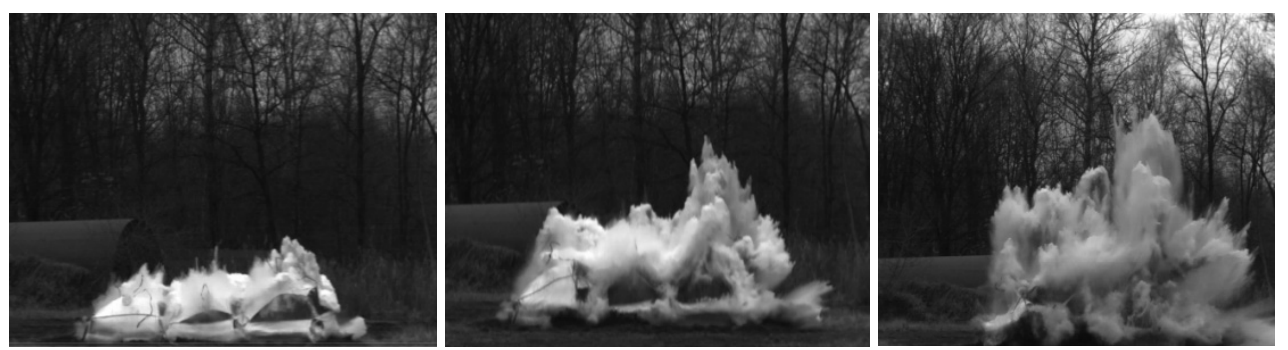

Figure 4: Time sequence of explosion of three water-bags placed next to each other. 


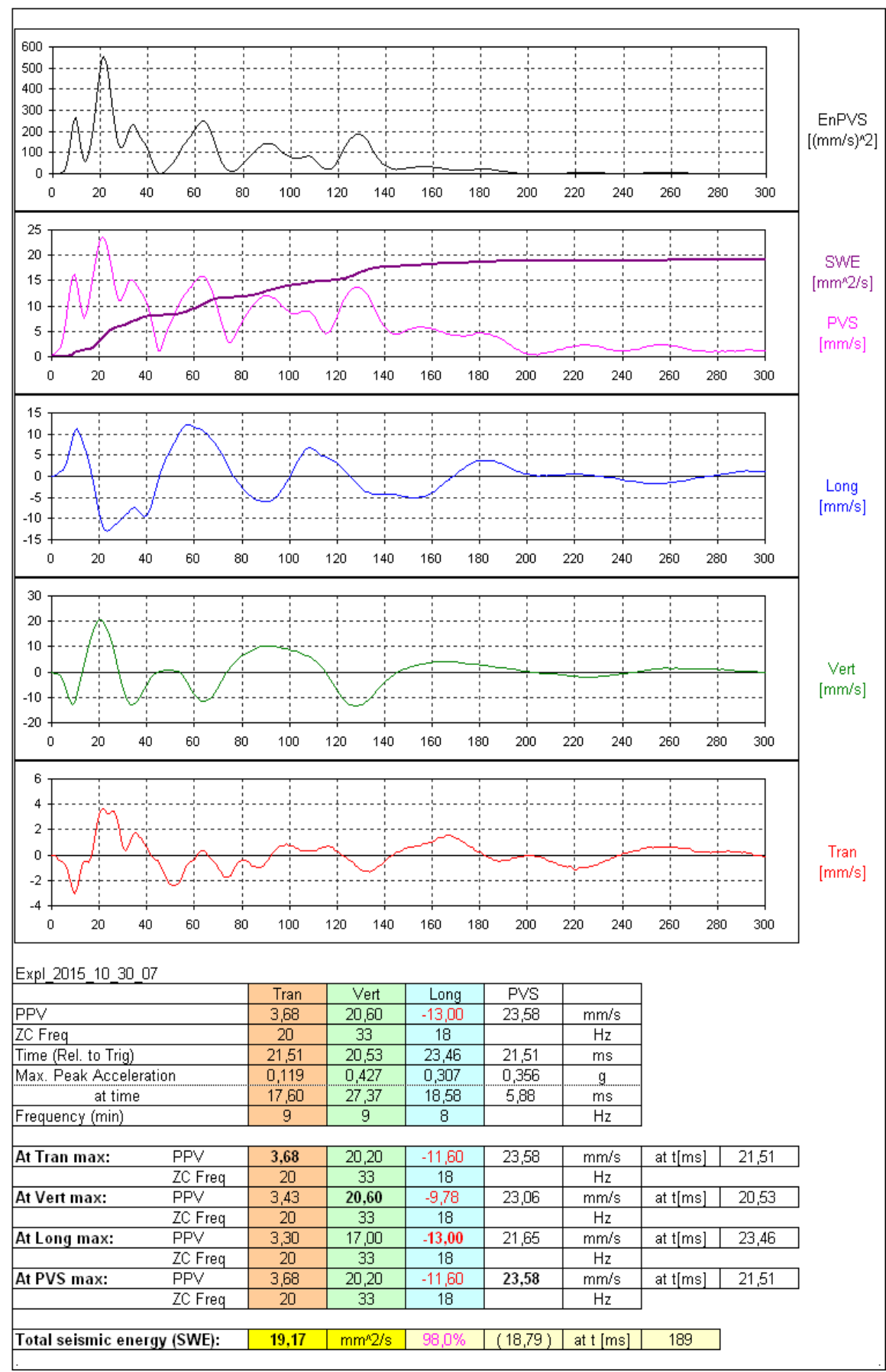

Figure 5: Measured data - spacing of explosive charges 1 and $2 \mathrm{~m}$, sequential timing $2 \mathrm{~ms}$. 


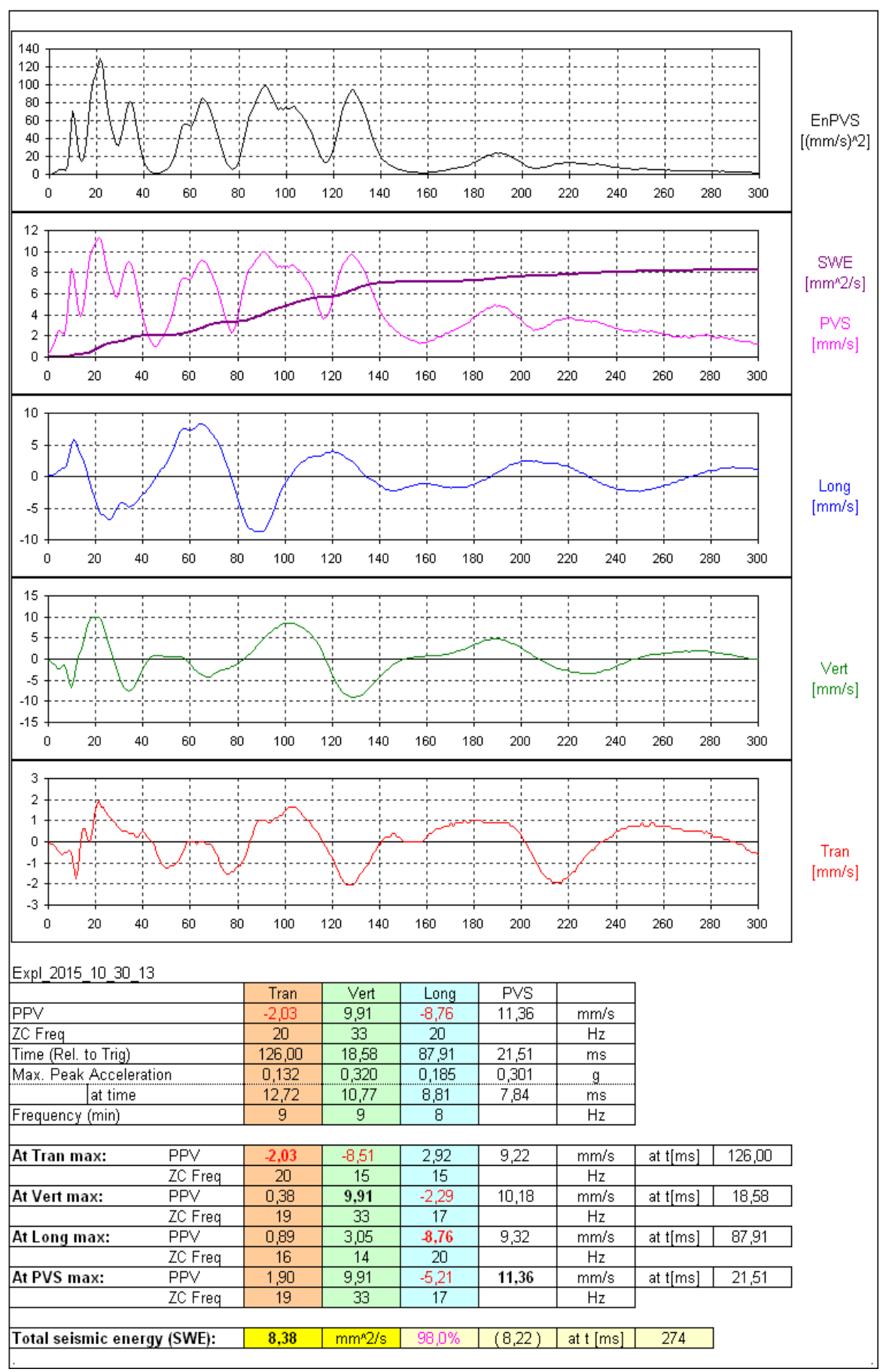

Figure 5: Continued Measured data - spacing of explosive charges 1 and $2 \mathrm{~m}$, sequential timing $2 \mathrm{~ms}$. 


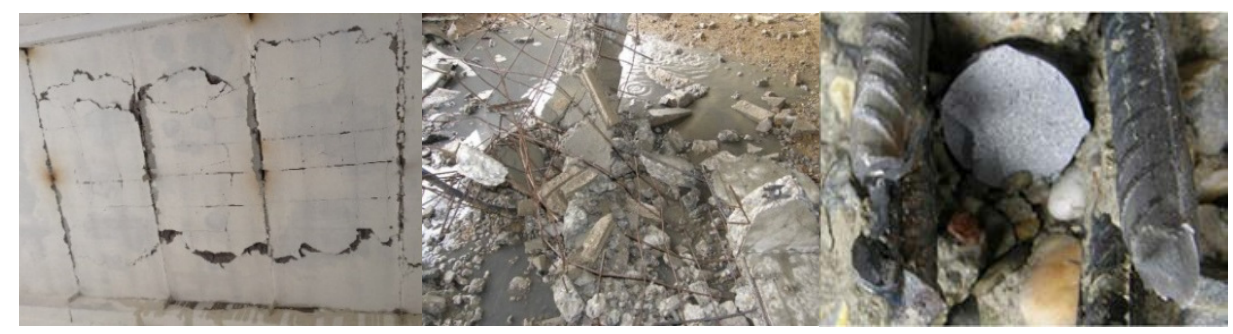

Figure 6: Practical tests on the reinforced-concrete floor of a prefabricated building.

Another practical realisation of tests was carried out on reinforced-concrete floors at simulated conditions in a scrapped storied prefab object, see Fig. 6.

Power of sequential increasing of energy at explosion of the two explosive charges next to each other is demonstrated in Fig. 6 (left) where huge cracks of the prefab floors occurred at detonation. Fig. 6 (middle) shows total breakthrough of the floor which happened at explosion when three explosive charges were next to each other. Detailed in Fig. 6 (right) presents damage of steel floor beams in the place of the performed experiment.

As mentioned above, this system of directed explosive charges could be used also for the rehabilitation of damaged buildings. Due to their dimensions, it is difficult to fix the textile sealing water-bags to the vertical walls. Therefore, the project of safety research was initiated for development of considerably smaller explosive charges working on the same principle as the already developed system of sequentially timing directed explosive charges.

\section{DEVELOPMENT OF SPECIAL DESTRUCTIVE EXPLOSIVE CHARGES}

Special destructive explosive charges should work on the principle of directivity of the working medium in the front part. By means of the explosive, this medium is cumulated to high-pressure flow of narrow cross-section. In the back part of the explosive charge, limited volume of water in "stuffing-box" is located. It provides a partly higher forwardly effect of the working jet and creates water mist in the back part of the explosive charge which prevents combustion of inflammable objects and also dampens the shock wave considerably in this zone. This avoids damage of surrounding objects and buildings in threatened area [1], [6].

For construction of special directed or cumulative explosive charges, the explosives with higher detonation velocity (more than $6000 \mathrm{~m} \cdot \mathrm{s}^{-1}$ ) are used, e. g. Hexogen $\left(8520 \mathrm{~m} \cdot \mathrm{s}^{-1}\right.$ ), Oktogen $\left(9100 \mathrm{~m} \cdot \mathrm{s}^{-1}\right)$, Semtex 1A, 1H, $10\left(7200,7400,7300 \mathrm{~m} \cdot \mathrm{s}^{-1}\right)$ or Semtex 10-SE $\left(7200 \mathrm{~m} \cdot \mathrm{s}^{-1}\right)$.

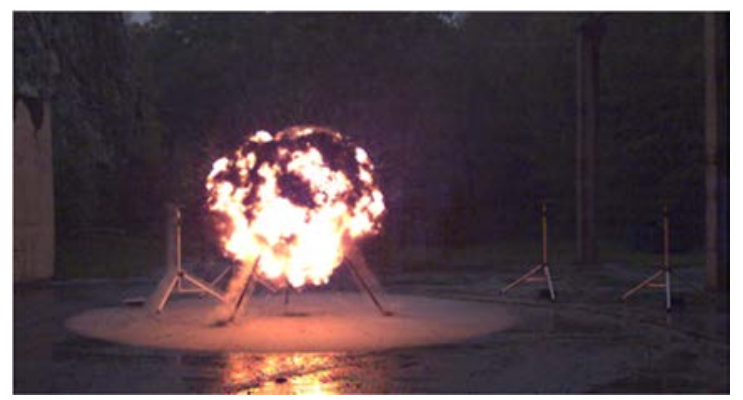

Figure 7: Test of uniformity of explosion of Semtex 10-SE, $t=5,2 \mathrm{~ms}$. 
Regarding easy handling with the plastic form of the explosive, it is advantageous to use the type Semtex 10-SE by Explosia A.S. company. It is provided in sheets of $2 \mathrm{~mm}$ thickness, $300 \mathrm{~mm}$ width and $10 \mathrm{~m}$ length. Its finish is adhesive and it can be placed in any shape of cumulative cavity. Its detonating ability is stable and uniform through whole diameter it is illustrated in Fig. 7 with a photo of the experimental test.

Graphs in Fig. 8 demonstrate courses and sizes of shock waves in defined distances from measured explosive samples - the epicentre of the explosion. Results correspond to Semtex $10-\mathrm{SE}$ with weight of $300 \mathrm{~g}$.

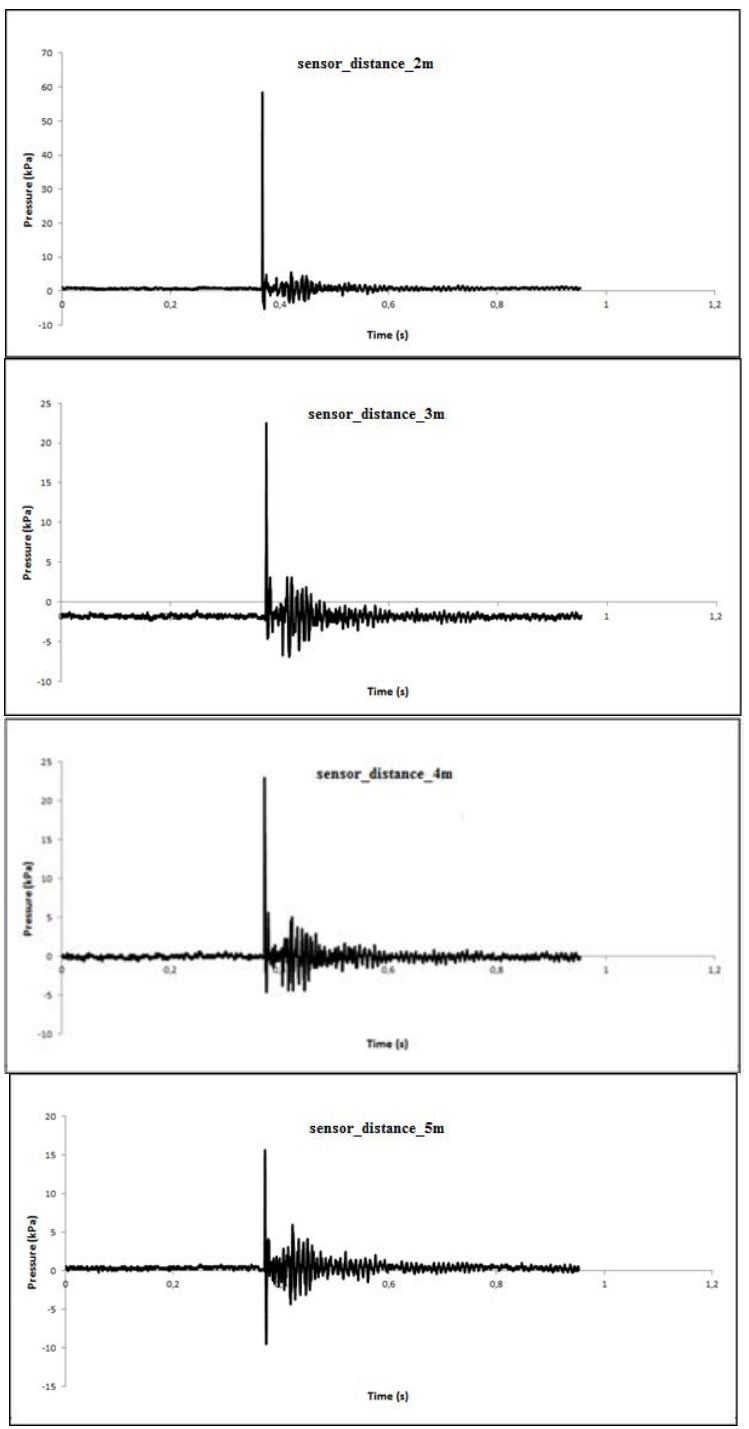

Figure 8: Courses of impulse waves of Semtex 10-SE, $300 \mathrm{~g}$. 
For verification of introduced theory of the directed explosive charge, its prototype was structurally designed for practical realisation of testing. Experiments should confirm whether sufficient cumulative pressure water jets arise for action on chosen structural elements of the walls. Tests should verify also the efficiency of water mass in the stuffing-box for elimination of a shock wave in the back part of the explosive charge. A plastic can was chosen as a package for the explosive charge. Its upper part was cut off so cumulative plastic insert with the explosive could be placed inside, see Fig. 9, where:

- No. 1 is a cumulative cavity for working water mass,

- No. 2 is the explosive of type Semtex 10-SE stuck to the cumulative cavity with detonator,

- No. 3 is the back part of the explosive charge for water mass which acts as a filling.

Fig. 10 then documents the origin of pressure water jet in the front part of the explosive charge were the velocity is c. $620 \mathrm{~m} / \mathrm{s}$.

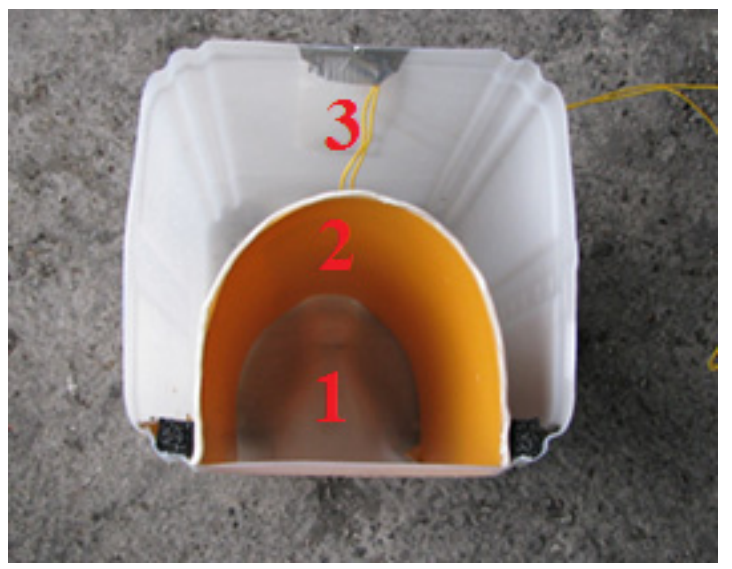

Figure 9: Construction of special directed explosive charge in plastic can.

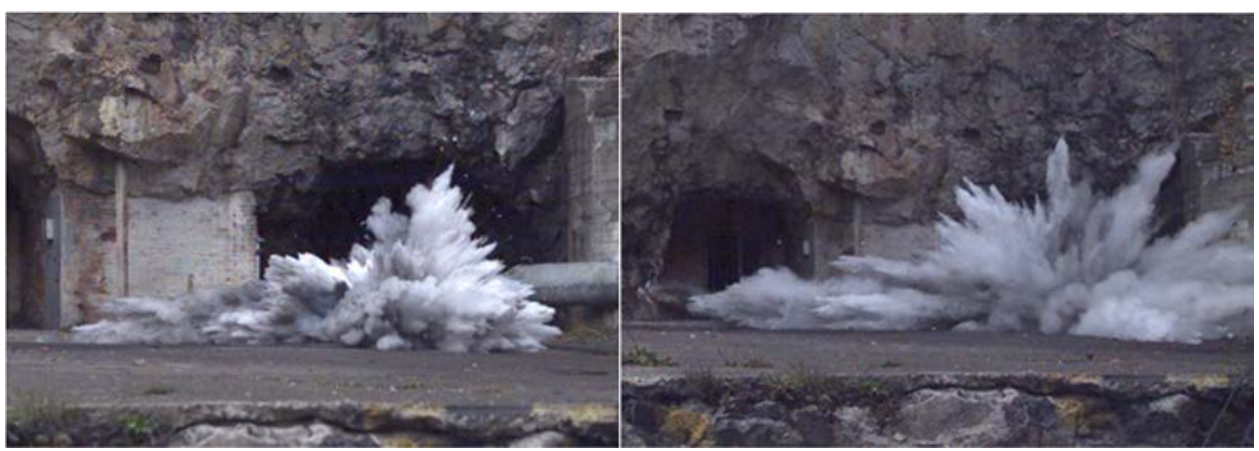

Figure 10: Test of special water explosive charge with total volume of $101, \mathrm{t}=0.6 \mathrm{~ms}$ (left) and $\mathrm{t}=1.5 \mathrm{~ms}$ (right). 


\section{CONCLUSIONS}

On the basis of the presented information, it could be stated that the development of special directed explosive charges and their practical implementation add another instrument for safe demolition of damaged buildings. Today, such instrument is absent among the equipment of the Fire Rescue Service which deal with rehabilitation of these buildings. Cumulative flow of working medium of developed charges arise from formation of the minimum amount of explosive and it is not the reason of bolstering fragmentation. It could also be used for creating a new evacuation exit in partly damaged buildings. In the cases of intensive fires, accidents involving gas pipes or pressure cylinders containing flammable gases, these developed charges will be tested within the experiments for the possibility of effective extinction of the flames epicentre by a huge water stream together with a blast wave of used operating explosives. A technical idea was inspired by experimental development of technology of non-invasive use of explosives at breaking ice flows in rivers. The system of special sequentially timed charges use the increase of efficiency of external charges and covering with water-bags together with the increase of efficiency of the whole system due to their timing. Timing, space combination at arrangement and jointing of the charges brings the effect of controlled release of the ice blocks in icebound water course and besides the regulation of floating ice size is possible as well. Developed charges increase the operability and safety of rescue units of IRS (Integrated Rescue System).

\section{ACKNOWLEDGEMENT}

This paper was financially supported by the project of grant ministry of interior the Czech Republic under the Id. No. VI20152019047, entitled "Development of the rescue destructive bombs for the disposal of statically damaged buildings".

\section{REFERENCES}

[1] Dojcar, O., Horky, J. \& Korinek, R., Blasting techniques (in Czech), Montanex, A.S., Ostrava 1996, ISBN 80-85780-69-0.

[2] Cooper, P.W. \& Kurovski, S.R., htroduction to the Technology of Explosives, VCHPubl., USA 1996.

[3] Lichorobiec, S. \& Barcova, K., Verification of the Efficacy of the Special Water Shaped Charge Prototype, Defence Science Journal, 65(5), Sep. 2015, pp. 363-366, 2015. DOI: $10.14429 / \mathrm{dsj} .65 .8850$

[4] Lichorobiec, S., Alternative development of projectiles to deactivate the explosive means explosive systems - pipe bombs. Communications, University of Žilina, pp. 2025, 2011. ISSN 1335-4205.

[5] Vavra, P. \& Vagenknecht, J., Theory of explosions, Pardubice University (in Czech), Faculty of Chemical Technology, 2004. ISBN 80-7194-494-7.

[6] Zukas, A.J. \& Walters, W.P., Explosive Effects and Applications, Springer-Verlag, N.Y. 1998. 